\title{
Expression of Myosin Heavy Chain Isoforms in Stimulated Fast and Slow Rat Muscles
}

\author{
Simonetta Ausoni, ${ }^{1}$ Luisa Gorza, ${ }^{1}$ Stefano Schiaffino, ${ }^{1}$ Kristian Gundersen, ${ }^{2,3}$ and Terje Lømo ${ }^{2}$ \\ IInstitute of General Pathology and CNR Unit for Muscle Biology and Physiopathology, University of Padova, Italy, \\ Institute of Neurophysiology, and Institute of Physiology, University of Oslo, 0162 Oslo 1, Norway
}

The expression of 4 myosin heavy chain (MHC) isoforms was analyzed in the rat soleus (SOL) and extensor digitorum longus (EDL) muscles after denervation and chronic electric stimulation. The stimulation frequencies used were 20 and $150 \mathrm{~Hz}$ and the amount of stimulation was either large (20 $\mathrm{Hz}$ ), intermediate $(150 \mathrm{~Hz})$, or small $(150 \mathrm{~Hz})$. These patterns resemble some features of normal motor unit activity in SOL and EDL of freely moving rats (Hennig and Lømo, 1985).

The relative expression of each MHC isoform depended strongly on the stimulation pattern. Furthermore, for any particular stimulation pattern, fibers in SOL and EDL expressed different MHCs. Coexistence of different MHC types in the same fiber was frequently observed in stimulated muscles. 20-Hz stimulation preserved normal expression of type 1-MHC in SOL but failed to induce type 1-MHC in type 2 fibers of the EDL, where type 2A- and $2 \mathrm{X}-\mathrm{MHC}$ expression dominated and type 2B-MHC expression was completely suppressed. 150-Hz low-amount stimulation preserved nearly normal 2BMHC expression in many type 2 fibers of the EDL but failed to induce type 2B-MHC expression in the SOL, where $2 \mathrm{X}$ MHC became predominant. 150-Hz high-amount stimulation differed from $150-\mathrm{Hz}$ small amount stimulation by suppressing almost all type 2B-MHC expression in EDL and by inducing considerable type 2A-MHC expression in the SOL. Scattered fibers in EDL that were probably the original type 1 fibers responded differently from both type 2 fibers in the EDL and from type 1 fibers in the SOL to stimulation.

The different MHCs expressed by these stimulation patterns corresponded well to the maximum intrinsic shortening velocities determined earlier in the same muscles (Eken and Gundersen, 1988). The incomplete slow-to-fast transformation of $V_{\max }$ in SOL can now be attributed to an inability of rat SOL muscles to express type 2B-MHC, whereas the incompletely fast-to-slow transformation of the rat EDL can be attributed to an inability of EDL type 2 fibers to express type 1-MHCs.

These results demonstrate the existence of intrinsic differences between SOL and EDL muscles in the rat and support the concept of different, but partially overlapping, adaptive ranges in these muscles (Westgaard and Lømo, 1988).

\footnotetext{
Received Apr. 3, 1989; revised June 19, 1989; accepted June 20, 1989.

We thank Massimo Fabbri, Maurizio Moretto, and Sigrid Schaller for technical assistance. This work was supported by CNR and Ministero della Pubblica Istruzione in Italy and by The Norwegian Research Council for Science and the Humanities.

Correspondence should be addressed to Stefano Schiaffino, Institute of General Pathology, Via Trieste 75, 35100 Padova, Italy.

Copyright $(\overline{\mathcal{C}} 1990$ Society for Neuroscience $0270-6474 / 90 / 010153-08 \$ 02.00 / 0$
}

Previous work has demonstrated that the contractile speed and metabolic properties of rat soleus (SOL) and extensor digitorum longus (EDL) muscles can be markedly affected by direct muscle stimulation (Lømo ct al., 1974; Ekcn and Gundersen, 1988; Gorza et al., 1988; Gundersen et al., 1988). In these experiments the stimulation was done on denervated muscles in order to exclude neurotrophic influences and to determine precisely the pattern of activity in the muscle. The experiments showed that stimulation patterns resembling normal EDL motor unit activity maintained essentially normal properties in the EDL and induced incomplete slow-to-fast transformation in the SOL, whereas stimulation patterns resembling normal SOL motor unit activity maintained essentially normal properties in the SOL and induced incomplete fast-to-slow transformation in the EDL. These results are similar to those obtained by cross-reinnervation of rat SOL and EDL muscles (Close, 1969; Gutmann and Carlson, 1975). The fast-to-slow transformation of the rat EDL differs from that observed in innervated fast muscles of the rabbit and the cat after tonic low-frequency stimulation by being less pronounced (Pette et al., 1973; Salmons and Sreter, 1976; Eerbeek et al., 1984).

In the present experiments, rat SOL and EDL muscles were denervated and stimulated for about 2 months with the patterns used in earlier experiments (Eken and Gundersen, 1988; Gundersen et al., 1988). These patterns have features that resemble normal SOL and EDL motor unit activities (Hennig and Lømo, 1985). We have previously shown that intermittent high-frequency stimulation of denervated rat SOL muscles induces the expression of type 2 myosin heavy chains (MHCs) as well as a doubling of intrinsic maximum shortening velocity (Gorza et al., 1988). In the present experiments we have made a more detailed analysis of MHC expression not only in the SOL, but also in the EDL.

The MHCs analyzed were types 1, 2A, and 2B, for which distinct genes have been identified (Izumo et al., 1986) and specific antibodies have been developed (Pierobon Bormioli et al., 1981). In addition, a recently discovered MHC, termed $2 \mathrm{X}$ (Schiaffino et al., 1986, 1989), was analyzed. This MHC, which probably corresponds to 1 of the 3 type 2 MHCs recently described by Bär and Pette (1988), is present in a large number of fibers in the normal rat diaphragm and in a smaller number of fibers in normal fast hind limb muscles and appears to be associated with an intermediate speed of shortening (Schiaffino et al., 1988).

\section{Materials and Methods}

Denervation and chronic stimulation. Young adult male Wistar rats, weighing 250-350 gm at the beginning of the experiment, were anes- 


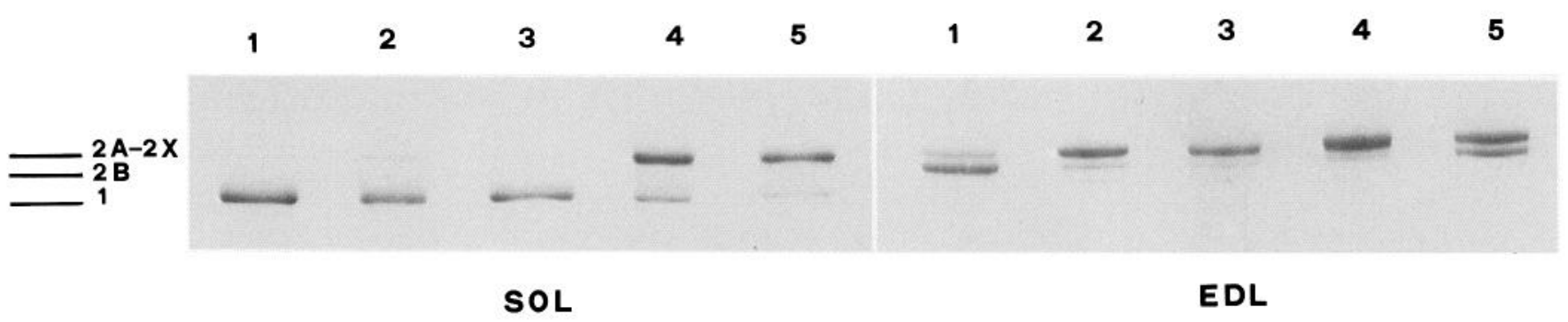

Figure 1. Electrophoretic analysis of MHC isoform expression in normal, denervated, and denervated-plus-stimulated EDL and SOL muscles. Three MHC bands were resolved in SDS-6\% polyacrylamide gels and stained with Coomassie blue. Only the region of the gel blots corresponding to the position of the MHCs is shown. Lane 1, control muscles; lane 2, denervated muscles; lane 3, muscles stimulated with the 20-Hz pattern; lane 4 , muscles stimulated with the $150-\mathrm{Hz}$ high-amount pattern; lane 5 , muscles stimulated with the $150-\mathrm{Hz}$ low-amount pattern.

thetized with Equithesin $(0.4 \mathrm{ml} / 100 \mathrm{gm}$ body weight i.p.). The denervation and stimulation procedures were essentially as described by Gorza et al. (1988). The sciatic nerve was cut and reflected in the thigh, and steel electrodes (AS 632, Cooner Sales Wire Co., Chatsworth, CA) were implanted on EDL or SOL muscles. Stimulation started 1-3 d after the operation and continued for 33-88 d (mean $63 \mathrm{~d}$ ). Contralateral innervated and denervated muscles were used as control. Three stimulation patterns were used: (1) 200 pulses at $20 \mathrm{~Hz}$ every $15 \mathrm{sec}$, (2) 25 pulses at $150 \mathrm{~Hz}$ every $15 \mathrm{sec}$ (" $150-\mathrm{Hz}$ high-amount"), and (3) 25 pulses at $150 \mathrm{~Hz}$ every $15 \mathrm{~min}$ ("150-Hz low-amount"; see Eken and Gundersen, 1988).

Electrophoretic and immunoblotting analysis. Procedures for myosin extraction, SDS-PAGE, and immunoblotting are described in detail elsewhere (Schiaffino et al., 1989). In brief, myosin was isolated from $20-30 \mathrm{mg}$ of tissue by a rapid method that involves first extraction of soluble proteins by a low-salt buffer and then extraction of myosin in

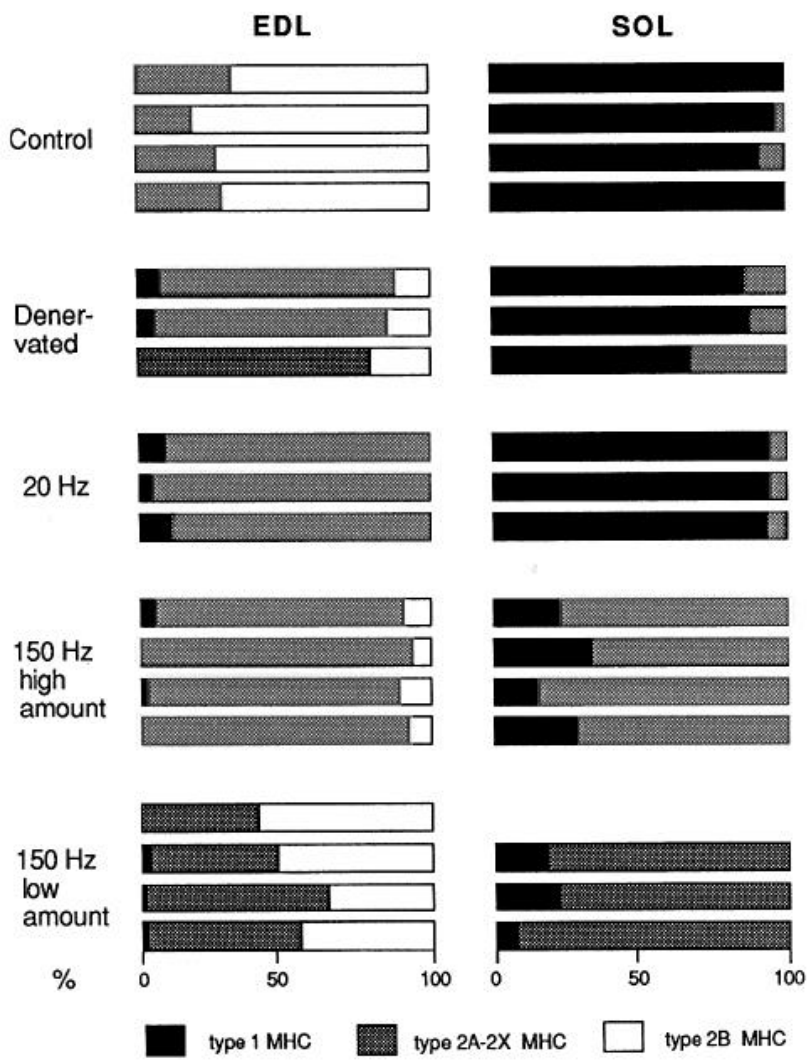

Figure 2. Relative proportions of type 1-, type 2A-2X-, and type 2BMHC bands based on quantitative densitometric measurements in Coomassie blue-stained SDS-6\% polyacrylamide gels of control, denervated, and stimulated muscles. Each bar corresponds to 1 muscle. a high-salt buffer. MHC isoforms were resolved in SDS-6\% polyacrylamide gels and stained with Coomassie blue. The gels were scanned with a Shimadzu CS-930 dual-wavelength TLC densitometer to estimate the relative amount of the MHC bands. For immunoblotting analysis proteins were electrophoretically transferred to nitrocellulose as described by Towbin et al. (1979). The blots were incubated with purified monoclonal antibodies and then with peroxidase-conjugated rabbit antimouse IgG antibodies, and peroxidase activity was revealed using diaminobenzidine as substrate.

Monoclonal antibodies. Monoclonal antibodies were raised in mice using myosin preparations from bovine or rat skeletal muscle as immunogen (Schiaffino et al., 1989). Selected hybridomas were cloned by limiting dilution and grown as ascites in pristane primed mice. Immunoglobulins were purified from ascites and their specificity for the different MHC isoforms was determined by immunoblotting. The following antibodies were used in this study: BF-32, which reacts with type 1- and 2A-MHC;RT-D9, which reacts with type 2X-and 2B-MHC; BF-D5, which reacts with type 1-MHC; SC-71, which reacts with type 2A-MHC;BF-F3, which reacts with type 2B-MHC; and SC-75, which reacts with type 2A-, 2X-, and 2B-MHCs (Schiaffino et al., 1989).

Immunohistochemistry. Serial transverse cryosections of control and stimulated muscles were incubated with appropriate dilutions of monoclonal antibodies and then with peroxidase-conjugated anti-mouse IgG antibodies, using standard procedures (Schiaffino et al., 1989).

\section{Results}

MHC isoforms expressed in normal, denervated, and stimulated muscle were identified by SDS-PAGE and by immunoblotting analysis with specific monoclonal antibodies. As previously reported (Schiaffino et al., 1988, 1989), 3 MHC bands can be resolved in Coomassie blue-stained gels: a high-mobility band corresponding to type 1-MHC, an intermediate-mobility band corresponding to type 2B-MHC, and a low-mobility band corresponding to type $2 \mathrm{~A}$ - and type $2 \mathrm{X}-\mathrm{MHCs}$ that comigrate in this electrophoretic system.

Figure 1 shows representative $\mathrm{MHC}$ bands from normal (lane 1), denervated (lane 2), or denervated and stimulated (lanes 35) EDL and SOL muscles. The relative amounts of MHC, as determined by densitometric analyses of Coomassie blue-stained gels in 3-4 muscles of each experimental group, are presented in Figure 2.

As shown in Figures 1 and 2, the normal EDL muscles contained large amounts of type 2B-MHC (mean 73.1\%) and smaller amounts of type $2 \mathrm{~A}-2 \mathrm{X}$ MHC $(26.8 \%)$, whereas the denervated EDL contained predominantly type $2 \mathrm{~A}-2 \mathrm{X}-\mathrm{MHC}(79.2 \%)$. Type $2 \mathrm{~A}-2 \mathrm{X}$ predominated also in EDL muscles stimulated with the $20 \mathrm{~Hz}(91.3 \%)$ and $150 \mathrm{~Hz}$ high-amount $(89.0 \%)$ pattern, whereas the EDL muscles stimulated with the $150 \mathrm{~Hz}$ lowamount pattern contained almost equal amounts of type 2B(45.2\%) and type $2 \mathrm{~A}-2 \mathrm{X}-(52.6 \%) \mathrm{MHCs}$. Interestingly, the slow, 
$2 A$

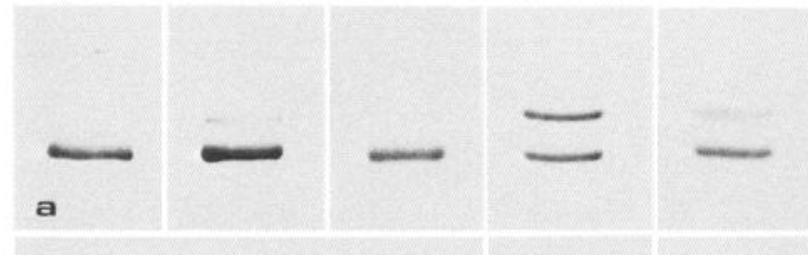

$2 X$

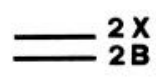

c

b

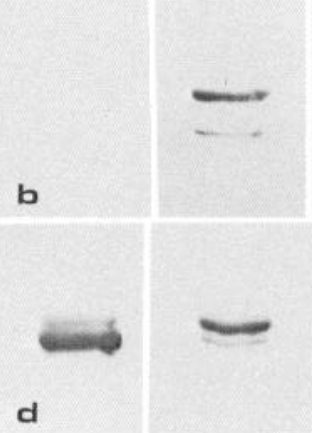

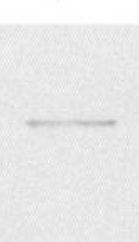

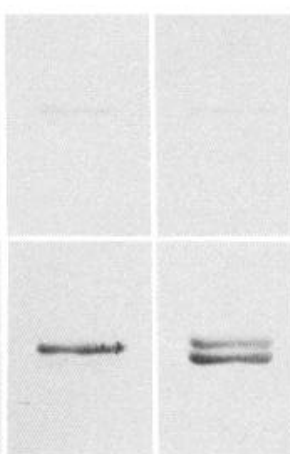

Figure 3. Immunoblotting analysis of MHC isoform expression in normal, denervated, and denervated-plus-stimulated EDL and SOL muscles. The myosin preparations shown in Figure 1 were transferred onto nitrocellulose and reacted with monoclonal antibodies specific for type 1- and 2A-MHCs $(a, b)$ or 2X-and 2B-MHCs $(c, d)$. Only the region of the blots corresponding to the position of MHCs is shown. Lane 1, control muscles; lane 2, denervated muscles; lane 3, muscles stimulated with the $20-\mathrm{Hz}$ pattern; lane 4 , muscles stimulated with the $150-\mathrm{Hz}$ high-amount pattern; lane 5 , muscles stimulated with the $150-\mathrm{Hz}$ low-amount pattern.

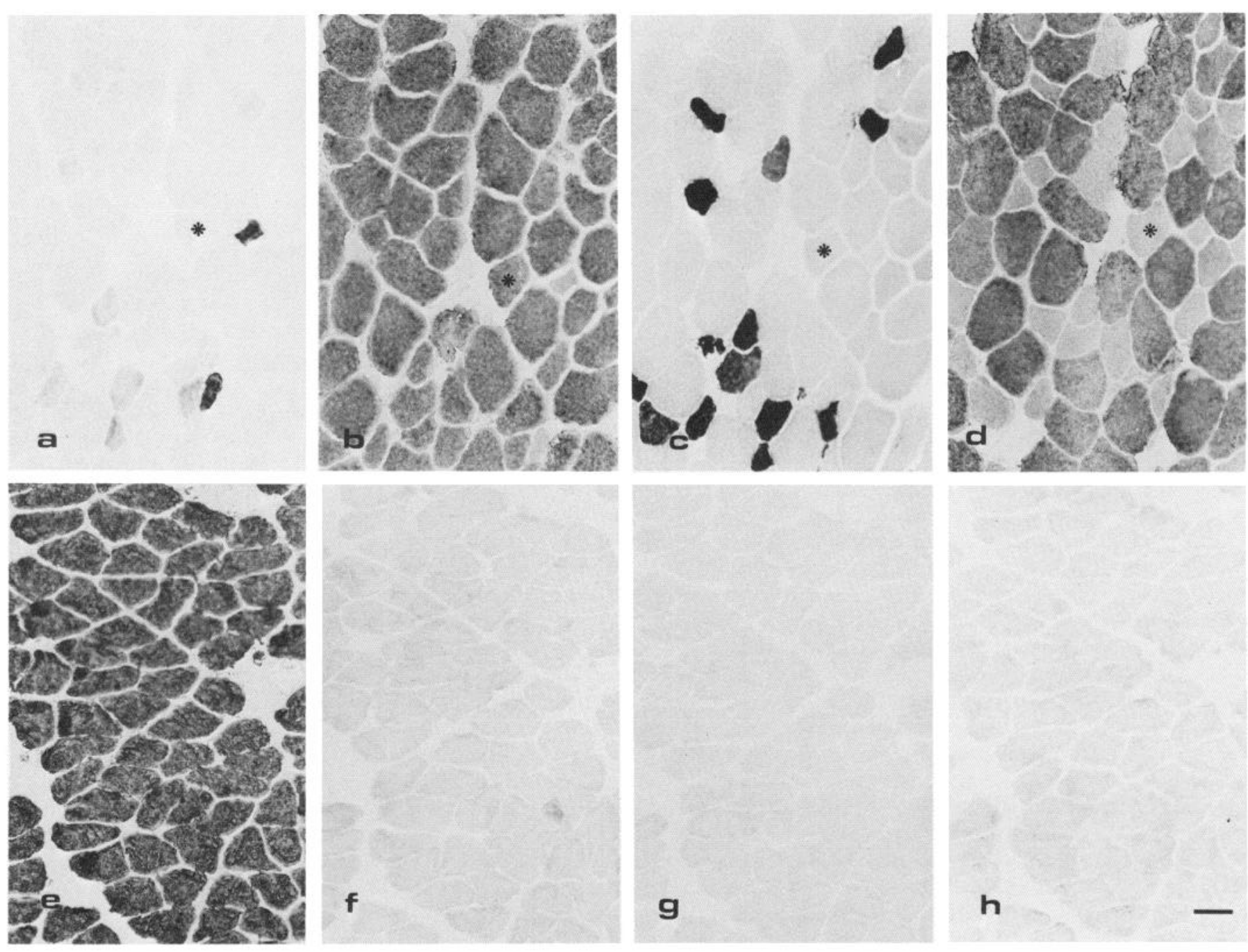

Figure 4. Immunoperoxidase staining of serial cryosections of control EDL $(a-d)$ and SOL $(e-h)$ with monoclonal antibodies specific for type 1-MHC $(a, e)$, all type $2 \mathrm{MHCs}(b, f)$, type 2A-MHC $(c, g)$, and 2B-MHC $(d, h)$. Asterisk marks a type $2 \mathrm{X}$ fiber, which stains for type 2-MHC but not for type $2 \mathrm{~A}$ - and $2 \mathrm{~B}-\mathrm{MHCs}$. 

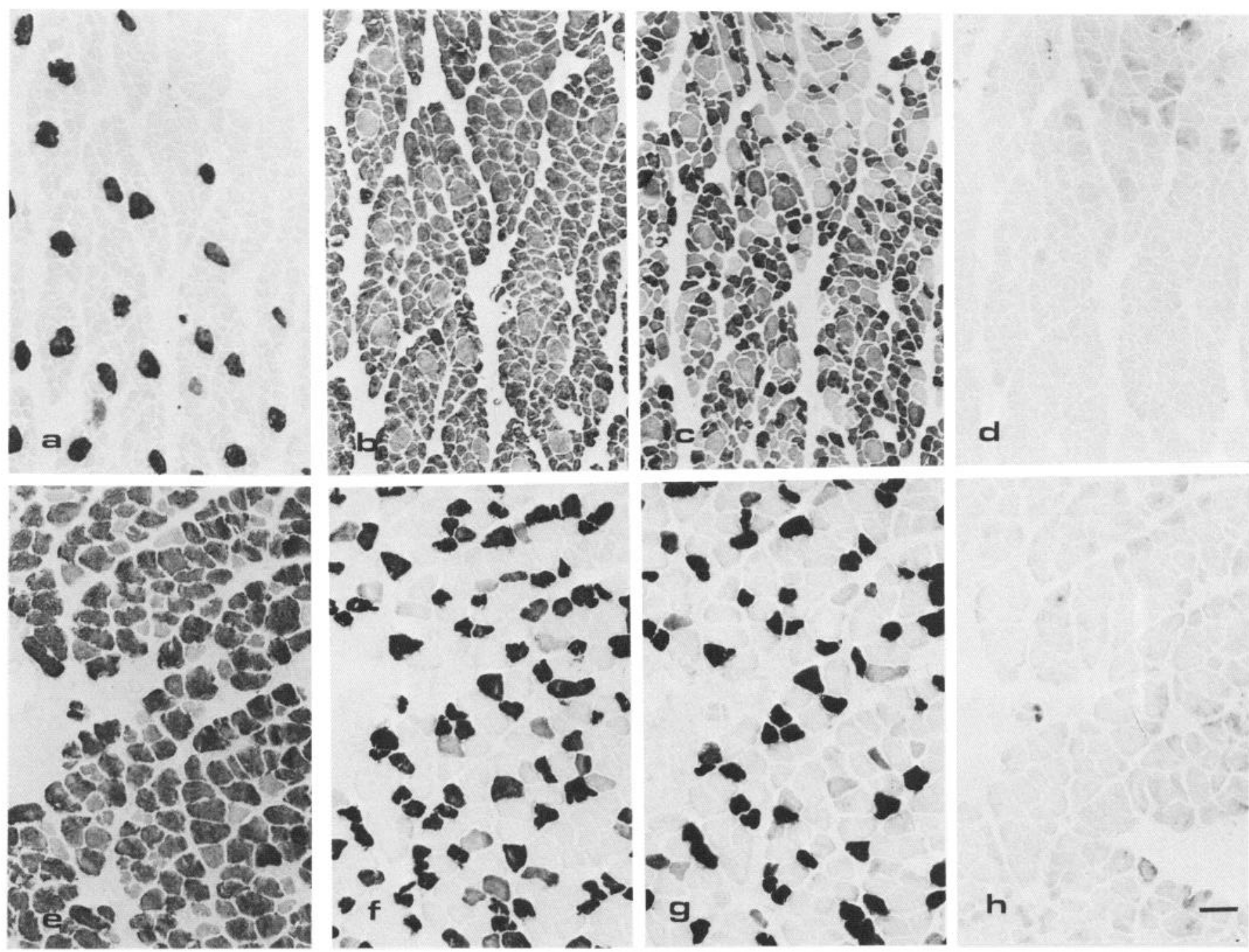

Figure 5. Immunoperoxidase staining of serial cryosections of denervated EDL $(a-d)$ and SOL $(e-h)$ with monoclonal antibodies specific for type 1-MHC $(a, e)$, all type 2 MHCs $(b, f)$, type 2A-MHC $(c, g)$, and 2B-MHC $(d, h)$.

20-Hz pattern suppressed all fast type 2B-MHC expression but failed to induce marked slow type 1-MHC expression (8.8\%).

The normal, denervated, and denervated plus $20 \mathrm{~Hz}$-stimulated SOL muscles contained predominantly type 1-MHC $(96.9$, 78.5 , and $93.7 \%$, respectively) and small amounts of type $2 \mathrm{~A}-$ 2X-MHCs. In contrast, SOL muscles stimulated at $150 \mathrm{~Hz}$ (highand low-amount) contained predominantly $2 \mathrm{~A}-2 \mathrm{X}-\mathrm{MHCs}(75.1$ and $84.3 \%$ ) and small amounts of type 1-MHC. Under no conditions did SOL muscles express type 2B-MHC.

To distinguish further between the different MHCs, such as those shown in Figure 1, the proteins were transferred to nitrocellulose and stained with monoclonal antibodies against type 1-2A-MHCs (Fig. 3a) and type 2X-2B-MHCs (Fig. 3b). As shown in Figure 3, 20- $\mathrm{Hz}$ stimulation induced only type 1-MHC expression in SOL and predominantly type 2X-MHC expression in EDL. In contrast, $150-\mathrm{Hz}$ high-amount stimulation induced roughly equal amounts of type $2 \mathrm{X}$ - and $2 \mathrm{~A}-\mathrm{MHCs}$ in SOL and predominantly $2 \mathrm{X}-\mathrm{MHC}$ in EDL, whereas $150-\mathrm{Hz}$-low amountstimulation induced predominantly type $2 \mathrm{X}$ - and some type $1-\mathrm{MHC}$ in SOL and approximately equal amounts of type $2 \mathrm{X}$ and 2B-MHCs in EDL.
The MHC composition of stimulated muscles was also investigated at the single-fiber level by immunoperoxidase staining of serial cryosections. A monoclonal antibody reactive with all 3 type 2-MHCs and monoclonal antibodies specific for type 1-, 2A-, and 2B-MHCs were used for this study. As shown in Figure 4, control SOL muscles were homogeneously composed of type 1 fibers, whereas control EDL muscles contained 3 subpopulations of type 2 fibers (2A, $2 \mathrm{X}$, and $2 \mathrm{~B})$ and rare type 1 fibers. Type $2 \mathrm{X}$ fibers were reactive for type 2-MHC, but unreactive for $2 \mathrm{~A}-$ and $2 \mathrm{~B}-\mathrm{MHCs}$. Fibers reacting for both type 1 - and $2 \mathrm{~A}-\mathrm{MHC}$ or both $2 \mathrm{~A}$ - and $2 \mathrm{X}-\mathrm{MHC}$ were very rare.

Denervation induced expression of $2 \mathrm{~A}-\mathrm{MHC}$ in many fibers in SOL and a striking decrease in 2B-MHC reactivity in most fibers in EDL (Fig. 5). Denervation atrophy affected all fibers in both muscles, except type 1 fibers in EDL. This finding may explain why type 1-MHC is detected in denervated but not in normal EDL by SDS-PAGE (see Figs. 1 and 3).

The $20-\mathrm{Hz}$ stimulation pattern maintained the normal histochemical fiber type profile in SOL and induced a complete disappearance of 2B-MHC in EDL (Fig. 6). In EDL, many type 2 fibers coexpressed type $2 \mathrm{~A}$ - and $2 \mathrm{X}-\mathrm{MHCs}$, while fibers ap- 


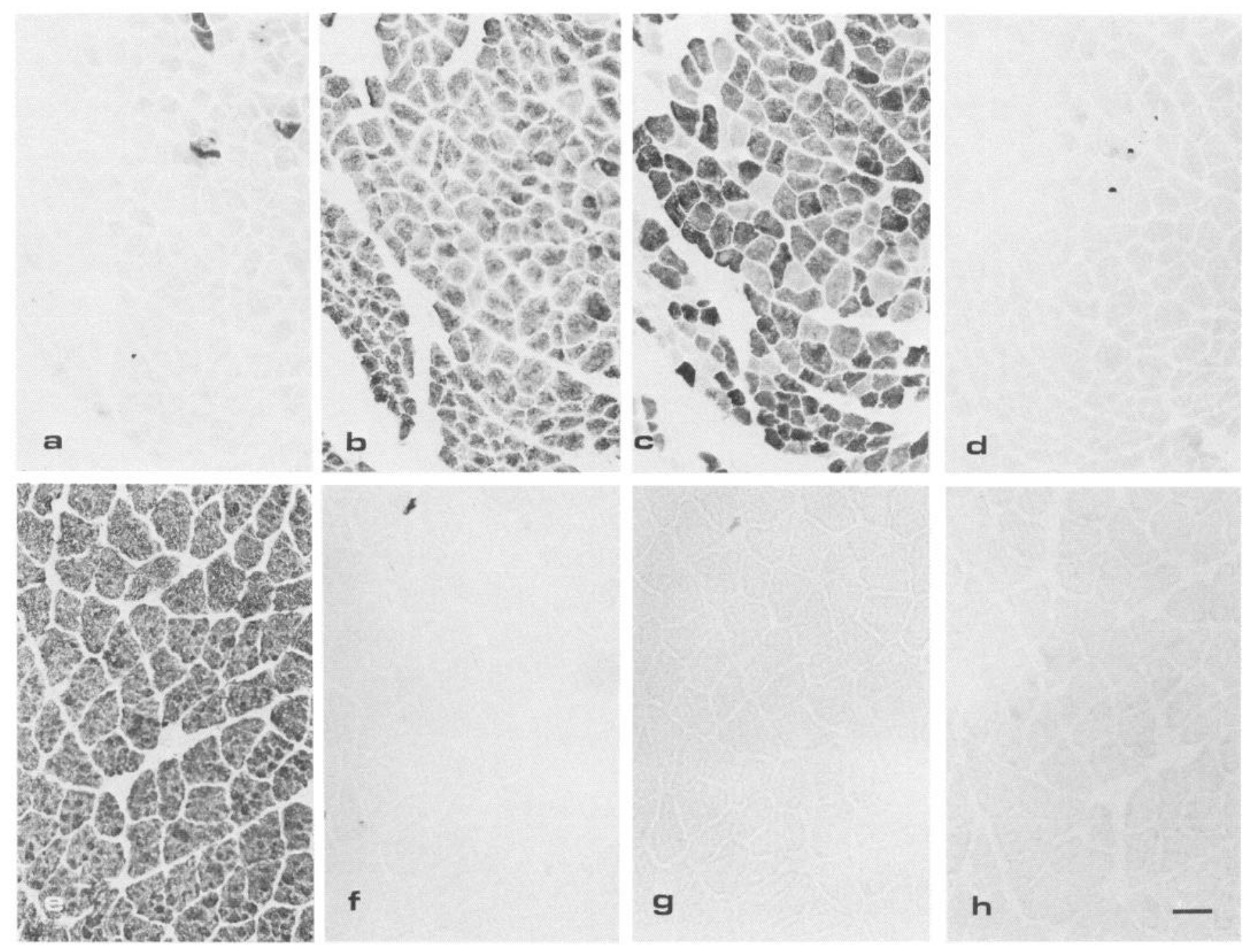

Figure 6. Immunoperoxidase staining of serial cryosections of 20-Hz-stimulated EDL $(a-d)$ an SOL $(e-h)$ with monoclonal antibodies specific for type $1-\operatorname{MHC}(a, e)$, all type $2 \mathrm{MHCs}(b, f)$, type $2 \mathrm{~A}-\mathrm{MHC}(c, g)$, and $2 \mathrm{~B}-\mathrm{MHC}(d, h)$.

parently corresponding to original type 1 fibers coexpressed type 1- and 2A-MHCs.

In EDL, the 150-Hz small-amount pattern preserve type 2BMHC expression in many fibers (Fig. 7). These muscles differed from normal, however, by having almost no type $2 \mathrm{~A}$ fibers, and by having a few, apparently original type 1 , fibers coexpressing type 1- and 2X-MHCs. In contrast, after stimulation with the $150-\mathrm{Hz}$ high-amount pattern, only very few fibers contained type $2 \mathrm{~B}-\mathrm{MHC}$, the rest expressing only type $2 \mathrm{X}-\mathrm{MHC}$ since they were reactive for type 2-MHC but unreactive for $2 \mathrm{~A}$ - and 2B-MHCs. Interestingly, a small number of fibers in both the $20-\mathrm{Hz}$ - and the $150-\mathrm{Hz}$-stimulated EDL muscles expressed type 1-MHC (Figs. 6 and 7). As these fibers had a distribution similar to that of type 1 fibers in the normal EDL (Fig. 4), they were probably original type 1 fibers. In addition, clusters of fibers staining for type 1-MHC were observed in some areas of some 20-Hz-stimulated EDL muscles.

In SOL, both stimulation patterns at $150 \mathrm{~Hz}$ induced type 2-MHC expression (Fig. 8). For the $150-\mathrm{Hz}$ low-amount pattern the expression was exclusively of the type $2 \mathrm{X}$ isoform as the fibers did not react with either anti-2A- or anti-2B-MHC an- tibodies. For the $150-\mathrm{Hz}$ high-amount pattern, however, there was a significant accumulation of $2 \mathrm{~A}-\mathrm{MHC}$. In addition, as previously described for SOL muscles stimulated at $100 \mathrm{~Hz}$ (Gorza et al., 1988; Schiaffino et al., 1988), most fibers retained significant reactivity for type 1-MHC. Three distinct MHCs, type 1-, 2A- and 2X-MHC, were thus coexpressed in most fibers in the $10-\mathrm{Hz}$ high-amount pattern group (Fig. $8, e-g$ ).

\section{Discussion}

Two main conclusions can be drawn from this work: first, that muscle stimulation strongly affects the expression of MHCs in rat SOL and EDL muscles, and second, that the same stimulus patterns result in different expressions of MHCs in SOL and EDL and apparently also in type 1 and type 2 fibers of the EDL. As MHC expression in striated muscle appears to mainly regulated at the transcriptional level (Izumo et al., 1986), these results indicate that MHC isogenes can be turned on and off by activity pattern in a muscle-type- and fiber-type-specific manner.

A major finding is that depending on the stimulation pattern, the SOL may express types 1-, 2A-, and 2X-, but not 2B-MHCs, 

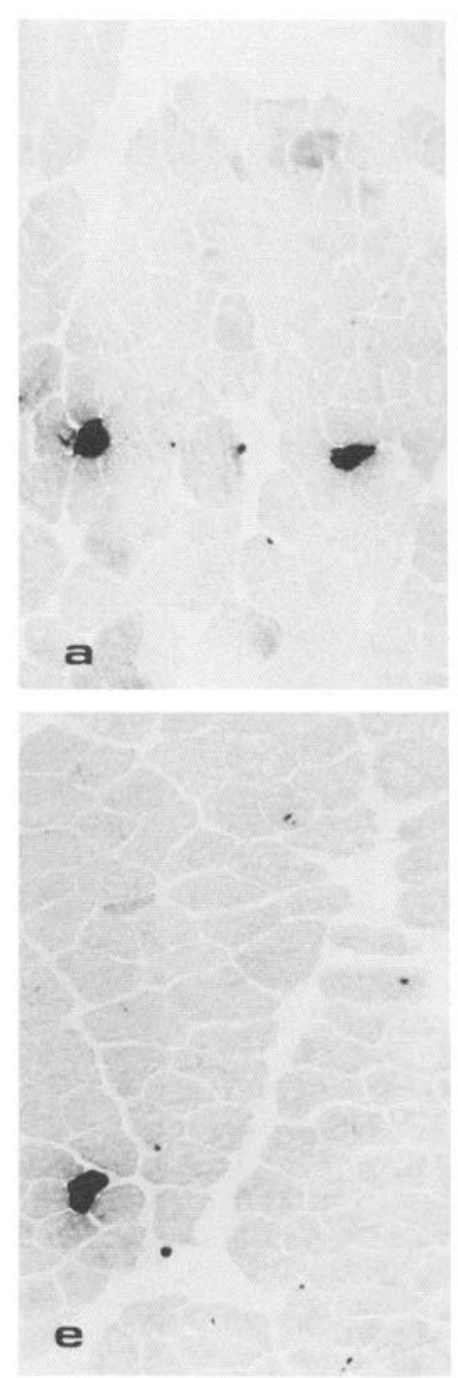
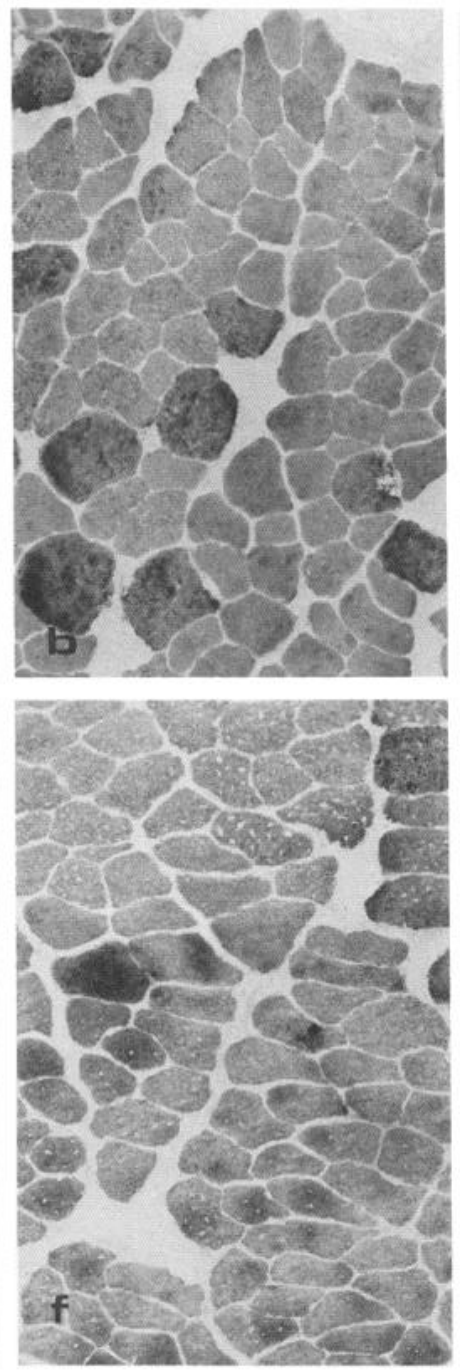
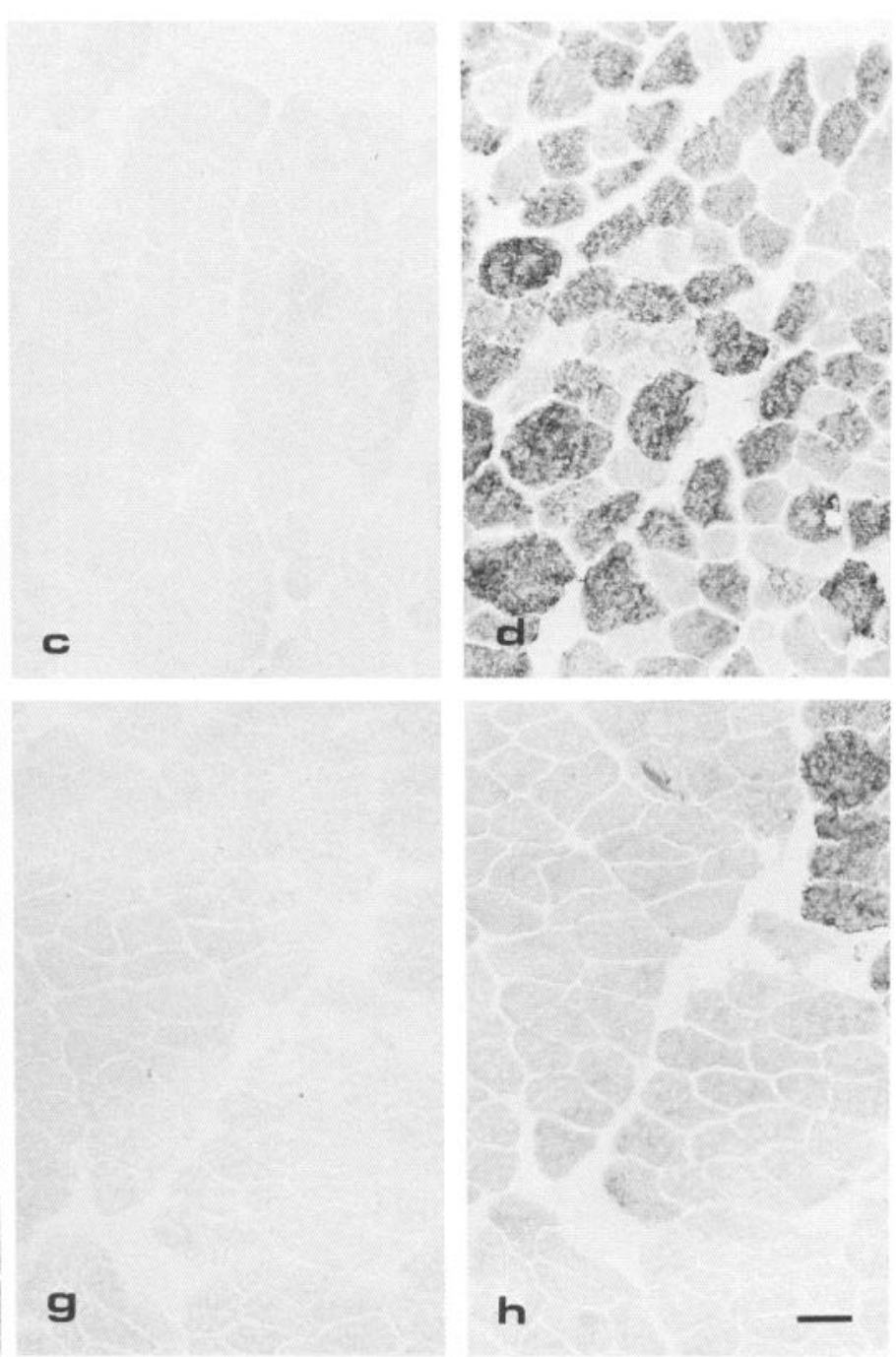

Figure 7. Serial cryosections of EDL stimulated with the $150-\mathrm{Hz}$ low-amount pattern $(a-d)$ and with the 150-Hz high-amount pattern $(e-h)$. Immunoperoxidase staining with monoclonal antibodies specific for type 1-MHC $(a, e)$, all type 2 MHCs $(b, f)$, type $2 \mathrm{~A}-\mathrm{MHC}(c, g)$, and $2 \mathrm{~B}-\mathrm{MHC}$ $(d, h)$.

whereas EDL type 2 fibers may express types 2B-, 2X-, and 2A-, but not 1-MHCs. This result suggests that tissue-specific differences between SOL and EDL render the gene coding for type 2B-MHC unavailable for activity-dependent expression in the SOL and the gene coding for type 1-MHC unavailable for activity-dependent expression in the EDL type 2 fibers.

In SOL, the expression of type $2 \mathrm{~A}-$ and $2 \mathrm{X}-\mathrm{MHCs}$ is turned off by $20-\mathrm{Hz}$ stimulation and turned on by $150-\mathrm{Hz}$ stimulation. The expression of type $2 \mathrm{~A}-\mathrm{MHC}$ is more pronounced during large amounts of $150-\mathrm{Hz}$ stimulation than during small amounts of $150-\mathrm{Hz}$ stimulation, whereas the converse is true for the expression of type $2 \mathrm{X}-\mathrm{MHC}$. This result indicates that large amounts of high-frequency activity up-regulate the expression of $2 \mathrm{~A}-\mathrm{MHC}$ and down-regulate the expression of $2 \mathrm{X}-\mathrm{MHC}$. Also, the expression of type 1-MHC can be graded by stimulation in the SOL. Thus, $94 \%$ of the MHCs expressed during $20-\mathrm{Hz}$ stimulation is of type 1 , whereas the corresponding mean percentages for the other stimulation patterns are $25 \%(150-\mathrm{Hz}$ high-amount) and 16\% (150-Hz low-amount).

In EDL, expression of type $2 \mathrm{~B}-\mathrm{MHC}$ is turned off by $20-\mathrm{Hz}$ stimulation, very weakly turned on by $150-\mathrm{Hz}$ high-amount, and strongly turned on by $150-\mathrm{Hz}$ low-amount stimulation. In addition, there is marked expression of $2 \mathrm{X}-\mathrm{MHC}$ and weak expression of 2A-MHC during $150-\mathrm{Hz}$ high-amount stimulation. In contrast, in the SOL the dominant effect of $150-\mathrm{Hz}$ high-amount stimulation is expression of 2A-MHC.

The different responses of SOL and EDL to identical stimulation point to intrinsically different properties in these fast and slow muscles. Similar intrinsic differences between rat SOL and EDL muscles have recently been demonstrated by thyroid hormone stimulation (Izumo et al., 1986). In addition, there appear to be intrinsic differences between type 1 and type 2 fibers in EDL. Thus, a few scattered fibers in the EDL, which probably correspond to the original type 1 fibers, express type 1-MHC during $150-\mathrm{Hz}$ stimulation, whereas the type 2 fibers do not. Assuming that these scattered fibers are the original type 1 fibers, then the type 1 fibers in the EDL also differ from the type 1 fibers in the SOL. For example, during $150-\mathrm{Hz}$ high-amount stimulation the type 1 fibers in EDL do not express type 2A$\mathrm{MHC}$, whereas in SOL they do. And during $20 \mathrm{-Hz}$ stimulation type 1 fibers in EDL express type 2A-MHC, whereas in SOL they do not. 

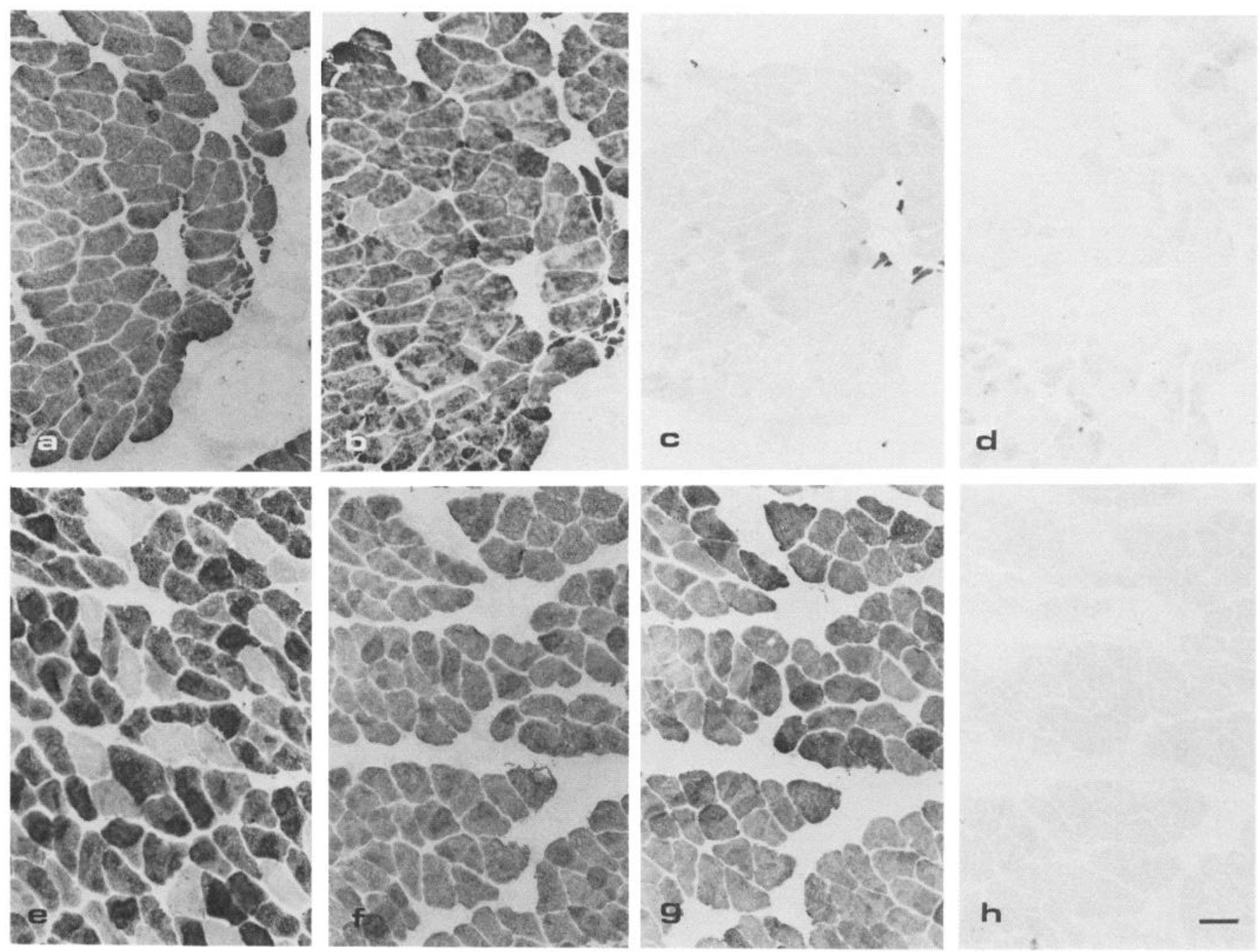

Figure 8. Serial cryosections of SOL stimulated with the $150-\mathrm{Hz}$ low-amount pattern $(a-d)$ and with the $150-\mathrm{Hz}$ high-amount pattern $(e-h)$. Immunoperoxidase staining with monoclonal antibodies specific for type 1-MHC $(a, e)$, all type 2 MHCs $(b, f)$, type $2 \mathrm{~A}-\mathrm{MHC}(c, g)$, and $2 \mathrm{~B}-\mathrm{MHC}$ $(d, h)$.

The coexistence of fast and slow myosin has previously been reported in transforming fibers of stimulated rabbit muscles (Rubinstein et al., 1978; Maier et al., 1988). This study shows that normal rat SOL and EDL muscles rarely contain more than 1 MHC. In contrast, stimulated muscle fibers often contained 2 , and in one case even 3, MHCs. These coexpressions were different in different fiber types and provide further evidence of intrinsic fiber differences and resistance to complete fiber type transformation.

Recent observations indicate that maximum shortening velocity at the sarcomere level (intrinsic $V_{\max }$ ) is determined by the type of MHC expressed in the muscle fiber (Reiser et al., 1985). There is also evidence that $V_{\max }$ of fibers expressing type 2A-MHC (Sweeney et al., 1986; Eddinger and Moss, 1987) or predominantly $(85 \%)$ type $2 \mathrm{X}-\mathrm{MHC}$ (Schiaffino et al., 1988) is intermediate between that of fibers expressing type 1-and type 2B-MHCs. The present results together with those of Eken and Gundersen (1988) are consistent with this conclusion. In control and $20-\mathrm{Hz}$-stimulated SOL muscles, which express predominantly type 1-MHC ( $99 \%$ and $94 \%$, respectively), $V_{\max }$ is about 5 fiber lengths per sec, whereas in control EDL muscles that express predominantly type 2B-MHCs $(68-81 \%), V_{\max }$ is about 15 fiber lengths per sec. Moreover, in the 20 - Hz-stimulated EDL muscles, which express predominantly the intermediate type $2 \mathrm{~A}-2 \mathrm{X}-\mathrm{MHCs}, V_{\max }$ is also intermediate $(11$ fiber lengths per sec). Thus, the incomplete fast-to-slow transformation of $V_{\max }$ in EDL by $20-\mathrm{Hz}$ stimulation can now be attributed to the inability of the EDL to express type 1-MHC and to the predominant expression of the intermediate types $2 \mathrm{~A}$ - and $2 \mathrm{X}$ MHCs. Similarly, the incomplete slow-to-fast transformation of $V_{\max }$ in high-frequency-stimulated SOL muscles can be attributed to the failure of the SOL to express type 2B-MHC as well as to the predominant expression of type 2A-2X-MHCs and a small residual expression of type 1-MHC.

Which aspect of a stimulation pattern is responsible for turning an MHC gene on or off is not very clear. The frequency of stimulation has a clear effect on twitch speed in rat SOL, but not in rat EDL or cat peroneus longus muscles (Kernell et al., 1987; Westgaard and Lømo, 1988; T. Eken and K. Gundersen, personal communication). Intrinsic $V_{\max }$ in SOL and EDL muscles of the rat appears to be regulated by amount rather than frequency of impulse activity (Eken and Gundersen, personal com- 
munication). In the tibialis anterior of the rabbit moderate amounts of stimulation transform type $2 \mathrm{~B}$ fibers to type $2 \mathrm{~A}$ fibers (Mabuchi et al., 1982), while larger amounts of stimulation result in transformation to atypical type 1 fibers (Staron et al., 1987). Also, differences in train duration appear to affect $V_{\max }$ (Eken and Gundersen, 1988) and MHC expression (our unpublished observation).

In conclusion, it appears that the properties of SOL and EDL in the rat can be graded by muscle activity within different adaptive ranges (Westgaard and Lømo, 1988). For the twitch contraction time the adaptive range extends from 12 to $45 \mathrm{msec}$ in the SOL and from 10 to $25 \mathrm{msec}$ in the EDL (Westgaard and Lømo, 1988). For intrinsic $V_{\max }$ the adaptive range, based on the relatively few stimulation patterns used so far, extends from 11 to 5 fiber lengths per sec in SOL and from 15 to 11 fiber lengths per sec in EDL (Eken and Gundersen, 1988). Finally, with respect to $\mathrm{MHC}$ expression, the SOL shows an adaptive range which includes type 1-, 2A-, and $2 \mathrm{X}$-, but not $2 \mathrm{~B}-\mathrm{MHCs}$, whereas EDL type 2 fibers show an adaptive range which includes type $2 \mathrm{~B}-, 2 \mathrm{X}-$, and $2 \mathrm{~A}-$, but not type 1 -MHCs. It will be of interest to investigate intermediate stages during the transformation of stimulated muscles to determine whether there is an obligatory scquence of MHC cxpression within these adaptive ranges.

\section{References}

Bär, A., and D. Pette (1988) Three fast myosin heavy chain in adult rat skeletal muscle. FEBS Lett. 235: 153-155.

Close, R. (1969) Dynamic properties of fast and slow skeletal muscles of the rat after nerve cross-union. J. Physiol. 204: 331-346.

Eddinger, T. J., and R. L. Moss (1987) Mechanical properties of skinned single fibers of identified types from rat diaphragm. Am. J. Physiol. 235: C210-C218.

Eerbeek, O., D, Kernell, and B. A. Verhey (1984) Effects of fast and slow patterns of tonic long-term stimulation on contractile properties of fast muscle in the cat. J. Physiol. 352: 73-90.

Eken, T., and K. Gundersen (1988) Chronic electrical stimulation resembling normal motor-unit activity: Effects on denervated fast and slow rat muscles. J. Physiol. 402: 651-669.

Gorza, L., K. Gundersen, T. Lomo, and S. Schiaffino (1988) Slow-tofast transformation of denervated soleus muscles by chronic highfrequency stimulation in the rat. J. Physiol. 402: 627-649.

Gundersen, K., E. Leberer, T. Lomo, D. Pette, and R. S. Staron (1988) Fibre types, calcium-sequestering proteins and metabolic enzymes in denervated and chronically stimulated muscles of the rat. J. Physiol. 398: 177-189.

Gutmann, E., and B. M. Carlson (1975) Contractile and histochemical properties of regenerating cross-transplanted fast and slow muscles in the rat. Pflüger's Arch. 353: 227-239.

Hennig, R., and T. Lomo (1985) Firing patterns of motor units in normal rats. Nature $314: 164-166$.

Izumo, S., B. Nadal-Ginard, and V. Mahdavi (1986) All members of the myosin heavy chain multi-gene family respond to thyroid hor- mone in a highly tissue specific manner. Science 231: 597-600.

Kernell, D., O. Eerbeek, B. A. Verhey, and Y. Donselaar (1987) Effects of physiological amounts of high- and low-rate chronic stimulation on cat's fast muscle. 1. Speed- and force-related properties. J. Neurophysiol. 58: 598-613.

Lømo, T., R. H. Westgaard, and H. A. Dahl (1974) Contractile properties of muscle: Control by pattern of muscle activity in the rat. Proc. R. Soc. Lond. (Biol.) 187: 99-103.

Mabuchi, K., D. Szvetko, K. Pinter, and F. A. Sreter (1982) Type IIB to IIA fiber transformation in intermittently stimulated rabbit muscles. Am. J. Physiol. 242: C373-C381.

Maier, A., L. Gorza, S. Schiaffino, and D. Pette (1988) A combined histochemical and immunohistochemical study on the dynamics of fast-to-slow fiber transformation in chronically stimulated rabbit muscle. Cell Tissue Res. 254: 59-68.

Pette, D., M. E. Smith, H. W. Staudte, and G. Vrbová (1973) Effects of long-term electrical stimulation on some contractile and metabolic characteristics of fast rabbit muscles. Pflüger's Arch. 338: 252-272.

Pierobon Bormioli, S., S. Sartore, L. Dalla Libera, M. Vitadello, and S. Schiaffino (1981) "Fast" isomyosins and fiber types in mammalian skeletal muscle. J. Histochem. Cytochem. 29: 1179-1188.

Reiser, P. J., R. L. Moss, G. G. Giulian, and M. L. Greaser (1985) Shortening velocity in single fibers from adult rabbit soleus muscles is correlated with myosin heavy chain composition. J. Biol. Chem. 260: 9077-9080.

Rubinstein, N., K. Mabuchi, F. Pepe, S. Salmons, J. Gergely, and F. Sreter (1978) Use of type-specific antimyosins to demonstrate the transformation of individual fibers in chronically stimulatcd rabbit fast muscles. J. Cell Biol. 79: 252-261.

Salmons, S., and F. A. Sreter (1976) Significance of impulse activity in the transformation of skeletal muscle type. Nature 263: 30-34.

Schiaffino, S., L. Saggin, A. Viel, S. Ausoni, S. Sartore, and L. Gorza (1986) Muscle fiber types identified by monoclonal antibodies to myosin heavy chains. In Biochemical Aspects of Physical Exercise, G. Benzi, L. Packer, and N. Siliprandi, eds., pp. 27-34, Elsevier, Amsterdam.

Schiaffino, S., S. Ausoni, L. Gorza, L. Saggin, K. Gundersen, and T. Lomo (1988) Myosin heavy chain isoforms and velocity of shortening of type 2 skeletal muscle fibres. Acta Physiol. Scand. 134: 575576.

Schiaffino, S., L. Gorza, S. Sartore, L. Saggin, S. Ausoni, M. Vianello, K. Gundersen, and T. Lomo (1989) Threc myosin heavy chain isoforms in type 2 skeletal muscle fibres. J. Muscle Res. Cell Motil. 10: 197-205.

Staron, R. S., B. Gohlsch, and D. Pette (1987) Myosin polymorphism in single fibers of chronically stimulated rabbit fast-twitch muscle. Pflüger's Arch. 408: 444-450.

Sweeney, H. L., M. J. Kushmerick, K. Mabuchi, J. Gergely, and F. A. Sreter (1986) Velocity of shortening and myosin isozymes in two types of rabbit fast-twitch muscle fibers. Am. J. Physiol. 251: C431C434.

Towbin, H., T. Staehelin, and J. Gordon (1979) Electrophoretic transfer of proteins from polyacrylamide gels to nitrocellulose sheets: Procedure and some applications. Proc. Natl. Acad. Sci. USA 76: 43504354.

Wcstgaard, R. H., and T. Lømo (1988) Control of contractile properties within adaptive ranges by patterns of impulse activity in the rat. J. Neurosci. 8: 4415-4426. 